\title{
Betreff Cadamuro et al.: In-vitro hemolysis and its financial impact using different blood collection systems
}

\author{
Regarding Cadamuro et al.: In-vitro hemolysis and its financial impact using different blood \\ collection systems
}

DOI 10.1515/labmed-2015-0101

Eingang 11.11.2015; Akzeptanz 9.12.2015

Schlüsselwörter: Blutabnahme; Hämolyse; Präanalytik.

Keywords: blood collection; hemolysis; preanalytics.

In ihrem Artikel "In-vitro hemolysis and its financial impact using different blood collection systems" stellen die Autoren Cadamuro et al. [1] ein sehr interessantes und praxisrelevant erhobenes Datenset dar und werten dieses nach relevanten Gesichtspunkten aus. Folgende drei Punkte wären eine interessante Ergänzung und wären mit hoher Wahrscheinlich aus dem vorliegenden Datenset zu ermitteln.

1. Die Autoren haben die Daten der Notaufnahme auf Grund mangelnder Vergleichbarkeit der Abnahmebedingungen ausgeschlossen, was grundsätzlich für eine komplette vergleichende Bewertung der Probenmaterialien Sinn mach, da die Abnahmebedingungen der Notaufnahme oftmals schlecht sind und die Probenqualität damit leidet. Eine separate Darstellung der Ergebnisse der verschiedenen Probenmaterialien auch in der Notaufnahme würde aber deswegen Sinn machen, da in einem Krankenhaus gerade von dort ein hoher und diagnostisch relevanter Probenanteil kommt.

2. Die Autoren verwenden für die Messung der Hämolyse einen klinisch chemischen Analyzer (COBAS, Roche, Switzerland). Da auf diesen Geräten meistens verschiedene Probenmaterialien untersucht werden, wie z.B. Serum und Material mit Glykolysehemmer,

*Korrespondenz: Dr. med. Alexander von Meyer, MBA, Chefarzt Laboratoriumsmedizin, Kliniken Nordoberpfalz AG, Klinikum Weiden, Söllnerstr. 16, 92637 Weiden, Germany, Tel.: +49 (0)961303 4500, Fax: +49 (0)961303 4506, E-Mail: alexander.vonmeyer@kliniken-nordoberpfalz.ag etc. können diese Werte prinzipiell auch zur Verfügung stehen. Da verschiedene Probenmaterialien unterschiedliche Hämolyseraten aufweisen können, ist eine Darstellung dieser Unterschiede in diesem speziellen Setting auch sehr interessant.

3. Die Autoren diskutieren einen Cut-Off für die Hämolyse um eine Probe als hämolytisch zu kennzeichnen. Auf Grund der heute existierenden exakten quantitativen Messmethoden für Interferenzen auf klinisch chemischen Analyzer und den Möglichkeiten modernen LIS-Systeme bietet es sich an, dass die Bewertung der Hämolyse quantitativ und testspezifisch erfolgt. Hiermit würden bei einem bestimmten Hämolysegrad z.B. eine LDH bereits als hämolytisch markiert sein, wobei andere Parameter noch ohne Markierung berichtet werden können. Eine derartige Bewertung des Datensets würde realistischer auch die entstehenden Zusatzkosten definieren lassen.

Abschließend ist zu hoffen, dass auch durch die vorliegende Arbeit der Wert der automatisierten Bewertung von präanalytischen Auffälligkeiten sowohl für Hersteller, als auch für Anwender einen höheren Stellenwert erhält und damit die Qualität durch einfachere Überwachung und nachfolgende auch die Kosten positiv beeinflusst werden.

Autorenbeteiligung: Der Autor trägt Verantwortung für den gesamten Inhalt dieses Artikels.

Forschungsförderung: Keine.

Interessenkonflikt: Kein Interessenkonflikt.

\section{Literatur}

1. Cadamuro J, Fiedler GM, Mrazek C, Felder TK, Oberkofler H, Kipman U, et al. In-vitro hemolysis and its financial impact using different blood collection systems. J Lab Med 2016;40:49-55. 